\title{
HUMAN VOMERONASAL EPITHELIUM DEVELOPMENT: AN IMMUNOHISTOCHEMICAL OVERVIEW
}

\author{
LÓRÁND DÉNES ${ }^{1}, Z_{\text {SUZSANNA PAP }}^{1}$, ANNAMÁRIA SZÁNTÓ ${ }^{1}$, \\ ISTVÁN GERGELY ${ }^{*}$ and TUdOR SORIN POP ${ }^{2}$ \\ ${ }^{1}$ Department of Anatomy and Embryology, University of Medicine and Pharmacy, \\ Tîrgu Mureş, Romania \\ ${ }^{2}$ Department of Orthopaedic Surgery and Traumatology I, \\ University of Medicine and Pharmacy, Tîrgu Mureş, Romania
}

(Received: 8 December 2014; accepted: 3 March 2015)

The vomeronasal organ (VNO) is the receptor structure of the vomeronasal system (VNS) in vertebrates. It is found bilaterally in the submucosa of the inferior part of the nasal septum. There are ongoing controversies regarding the functionality of this organ in humans. In this study we propose the immunohistochemical evaluation of changes in components of the human vomeronasal epithelium during foetal development. We used 45 foetuses of different age, which were included in three age groups. After VNO identification immunohistochemical reactions were performed using primary antibodies against the following: neuron specific enolase, calretinin, neurofilament, chromogranin, synaptophysin, cytokeratin 7, pan-cytokeratin and S100 protein. Digital slides were obtained and following colorimetric segmentation, surface area measurements were performed. The VNO was found in less than half of the studied specimens (42.2\%). Neuron specific enolase and calretinin immunoexpression showed a decreasing trend with foetal age, while the other neural/neuroendocrine markers were negative in all specimens. Cytokeratin 7 expression increased with age, while Pan-Ctk had no significant variations. S100 protein immunoexpression also decreased around the VNO. The results of the present work uphold the theory of regression of the neuroepithelium that is present during initial stages of foetal development.

Keywords: human, foetal, vomeronasal organ, immunohistochemistry

* Corresponding author; E-mail: gergelyistvan@studium.ro 


\section{Introduction}

The existence of a vomeronasal organ (VNO) in the human embryo, similar to VNOs of other species is well established [1]. It contains bipolar cells similar to vomeronasal neurons of other species, and it also generates cells producing luteinizing hormone releasing hormone (LHRH) [2]. These authors also showed that the structure becomes more simplified with advancement of development. Kjaer and Fischer-Hansen [3] did not find any structure resembling the VNO in later stages (19 weeks), although other authors demonstrated the presence of a precarious $\mathrm{VNO}$, which continued to grow in size until the age of at least 30 weeks [4].

Literature data on the human VNO localizes the organ on the nasal septum, and there is a consensus that it is a blind diverticulum in the septal mucosa, opening towards the nasal cavity through a small groove (the vomeronasal fossa) found approximately $2 \mathrm{~cm}$ posterior to the nostril. Localization of this structure corresponds to VNO location in the embryo, [5] and it has a similar simplified shape, without large vessels, cavernous sinuses or supporting cartilage. Some reports describe the structure in over $90 \%$ of the subjects (at least unilaterally), while others report $50 \%$ or less frequency.

There are ongoing controversies regarding the functionality of the human VN system. The human VNO not only differs significantly from the VNO of other species, but also there are contradictory reports regarding its postnatal persistence or involution.

Also, it has been established that immunological development, differentiation and maturation of tissue components is time dependent in the developing organism, showing uneven distribution of time points marking the initial occurrence of characteristic epitopes for various cellular markers and the transformation of initial, partially differentiated cell types into different mature cells.

The aim of the immunohistochemical study of the human foetal VNO is to characterize this epithelial structure and gain insight into the time sequence of immunological development of its different cellular components. Given large number of reports with contradicting results and conclusions about the human $\mathrm{VNO}$, we considered the evaluation of the epithelial dynamics during foetal development of the human VNO, using immunohistochemistry.

\section{Materials and Methods}

We used 45 human foetuses of different age. The specimens were selected based on macroscopic evaluation with the single criterion of "normal" external 
morphology. The foetuses were fixed in $4 \%$ formalin solution for variable intervals, but not less than 5 days; also, the nasal cavities were injected manually through the nostrils with formalin solution.

Before dissection we measured crown-rump length (CRL) and head circumference $(\mathrm{HC})$ of each foetus in order to establish foetal age. The nasal septa were removed by macroscopic dissection and the obtained samples were oriented in the frontal plane and sectioned macroscopically. The resulting $4-5 \mathrm{~mm}$ thick fragments were paraffin embedded and 5 micrometer thick histological sections were obtained.

Following positive identification of the VNO we proceeded to immunohistochemistry performed at the Department of Anatomy and Embryology of the University of Medicine and Pharmacy, Tîrgu Mureş, Romania.

The antibody panel used for detection of different possible components of this epithelium has been prepared using strict criteria, and the changes in immunoexpression during fetal development were monitored by studying foetuses of different age. Our first aim was to clarify the most important and most debated feature of the human VN epithelium, i.e. the presence of intraepithelial nervous structures that might be regarded as chemoreceptor cells, analogous to the receptor cells of the VNO of other mammals. We used neuron specific enolase (NSE), calretinin (Calret), neurofilament (NF), chromogranin (CHR) and synaptophysin (SYN) for this purpose. To confirm the epithelial characteristics (and to indirectly track neural elements) we used cytokeratin 7 (Ctk7) and an anti-cytokera-

Table I. Primary antibodies used

\begin{tabular}{lllll}
\hline Antibody & Clone & Manufacturer & Antigen retrieval solution & Dilution \\
\hline Neuronal/neuroendocrine markers & & & \\
\hline NSE & $5 \mathrm{E} 2$ & Novocastra & Citrate 6 & $1: 75$ \\
Calretinin & 5A5 & Labvision & Citrate 6 & $1: 80$ \\
Neurofilament & NR4 & DAKO & Citrate 6 & $1: 50$ \\
Chromogranin & DAK-A3 & DAKO & None & $1: 100$ \\
Synaptophysin & SYPOL & Labvision & Citrate 6 & $1: 80$ \\
\hline Epithelial markers & & & & $1: 100$ \\
\hline Ctk7 Ab2 & OV-TL-12/30 & Labvision & EDTA & $1: 150$ \\
PanCtk & AE1/AE3 & Labvision & EDTA & $1: 150$ \\
\hline Glial markers & & & & Citrate 6 \\
\hline S100 Ab1 & 4C4.9 & Labvision & & \\
\hline
\end{tabular}


Table II. Age groups of the studied fetuses

\begin{tabular}{lll}
\hline & CRL $(\mathrm{cm})$ & Fetal age (weeks) \\
\hline Group 1 & $5-14$ & $9-16$ \\
Group 2 & $15-23$ & $17-24$ \\
Group 3 & $24-$ & $25-$ \\
\hline
\end{tabular}

tin antibody cocktail (pan-cytokeratin - PanCtk). Glial cells, as neuron-associated structures are important in the context of Schwann-cells around the possible connections of the VNO with the olfactory bulb; we used S100 protein for identification (Table I).

\section{Distribution of studied foetuses over the foetal period}

Considering the available material, we distributed the foetuses into three age groups that covered most of the foetal period. Establishment of foetal age was performed based on CRL measurements, according to Langman's Medical Embryology [6] (Table II).

\section{Processing of digital slides and result analysis}

Immunohistochemistry slides were scanned using a Zeiss MiraxScan digital slide acquisition system (Carl Zeiss Jena GmbH, Jena, Germany) mounted with a Marlin F-146C digital camera with a Sony ICX267 sensor (Allied Vision Technologies GmbH, Stadtroda, Germany) at the Pathology Department of the Clinical County Hospital of Tîrgu Mureş, Romania. The digital images were examined using the Pannoramic Viewer software, version 1.15.4, developed by 3DHISTECH Ltd.

For standardization purposes, capturing still images of the regions of interest (areas representing the VNO and its close proximity, and other relevant areas of the section) was performed using a magnification factor of $\times 40$. These captured images were used subsequently for quantification of the immunoexpression. Additionally, we performed still image capture with other zoom factors for visual representation of the results. The still images were saved as TIFF files.

Immunohistochemical changes with foetal age have been described using the captured images with a software developed for biomedical image analysis, called ImageJ (version 1.6.0_15). 
In order to quantify the intraepithelial IHC expression (where applicable) we calculated the area of positive reaction reported to the total cross-sectional surface area of the $\mathrm{VN}$ epithelium.

Using ImageJ we performed colorimetric segmentation, which resulted in delineation of the positive reaction surface area for the given marker. For each marker, we identified four standardized, square-shaped regions of interest on the VNO epithelium. These were fitted to the actual thickness of the VN epithelium in each case. The colour image has been transformed into a black and white image (B/W 8 bit), and the area of positive reaction has been identified. Using the "Measure" function (with adequate settings) we measured the ratio of positive reaction for each region of interest, and calculated a simple average to reflect the percent of surface area with positive reaction compared to the entire surface of the VNO epithelium. The values obtained for individual foetuses included in the same age group were finally averaged to obtain a final ratio characteristic to each group.

\section{Results}

The distribution of VNO positive and VNO negative foetuses, as well as gender distribution of the foetuses is summarized in Table III below.

It is to be noted that of the 45 foetuses, less than half (42.2\%) possesses a $\mathrm{VNO}$, suggesting that the organ is inconstant in humans. The method we used (serial sectioning) excludes the possibility of accidentally omitting an existing organ. In the studied specimens the gender ratio is 1.37 , favouring the male foetuses. Nevertheless, frequency calculations suggest that the presence of the VNO is independent of the gender of the foetus and foetal age, so the global ratio of $\mathrm{VNO}+$ and $\mathrm{VNO}-$ cases can be used for subsequent data collection.

Table III. Distribution of foetuses regarding presence of the VNO and gender

\begin{tabular}{lccc}
\hline & VNO + & VNO- & Total \\
\hline Male & 11 & 16 & 27 \\
Female & 8 & 10 & 18 \\
Total & 19 & 26 & 45 \\
\hline
\end{tabular}



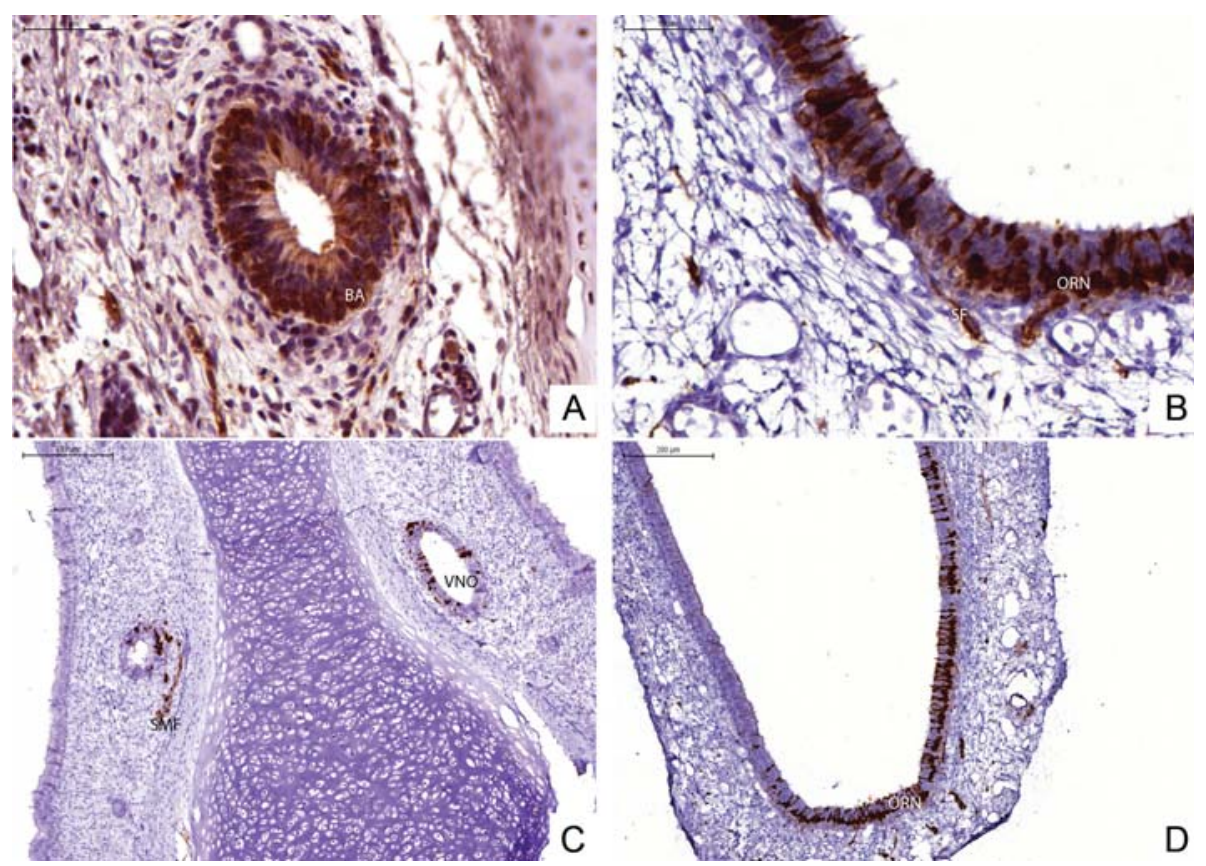

Figure 1. A) Neuron-specific enolase reaction in the VNO of the first age group, $\times 40$ magnification (BA - strong immunopositivity in the basal cell layer of the VNO).

B) Neuron-specific enolase reaction in the olfactory epithelium of the first age group, $\times 40$ magnification (ORN - positive receptor neurons, $\mathrm{SF}-$ positive subepithelial fibers).

C) Calretinin reaction in the VNO of the second age group, $\times 5$ magnification (VNO - location of the vomeronasal organ containing dispersed positive cells, SMF - positive submucosal fibres).

D) Calretinin reaction in the olfactory epithelium of the second age group, $\times 10$ magnification (note the consistent pattern of ORN reaching the luminal surface)

\section{Neuronal and neuroendocrine markers}

Neuron-specific enolase

In the first age group NSE positive cells are generally located in the basal layer of the VNO, encircling almost continuously the organ. There are a number of cells found in more superficial layers, as well as processes of basal cells that reach the luminal surface (Fig. 1A). The intermediate layers are clearly negative. Around the VNO there are fine nerve fibres highlighted by the immunohistochemical reaction. Examining the main olfactory epithelium for comparison, there is an intensively positive reaction exclusively confined to the bipolar cells that provide extensions consistently reaching the surface of the main olfactory epithelium, and basal extensions that leave the epithelium (Fig. 1B). Foetuses in the second age group show a decreasing amount of positive staining, with a dis- 


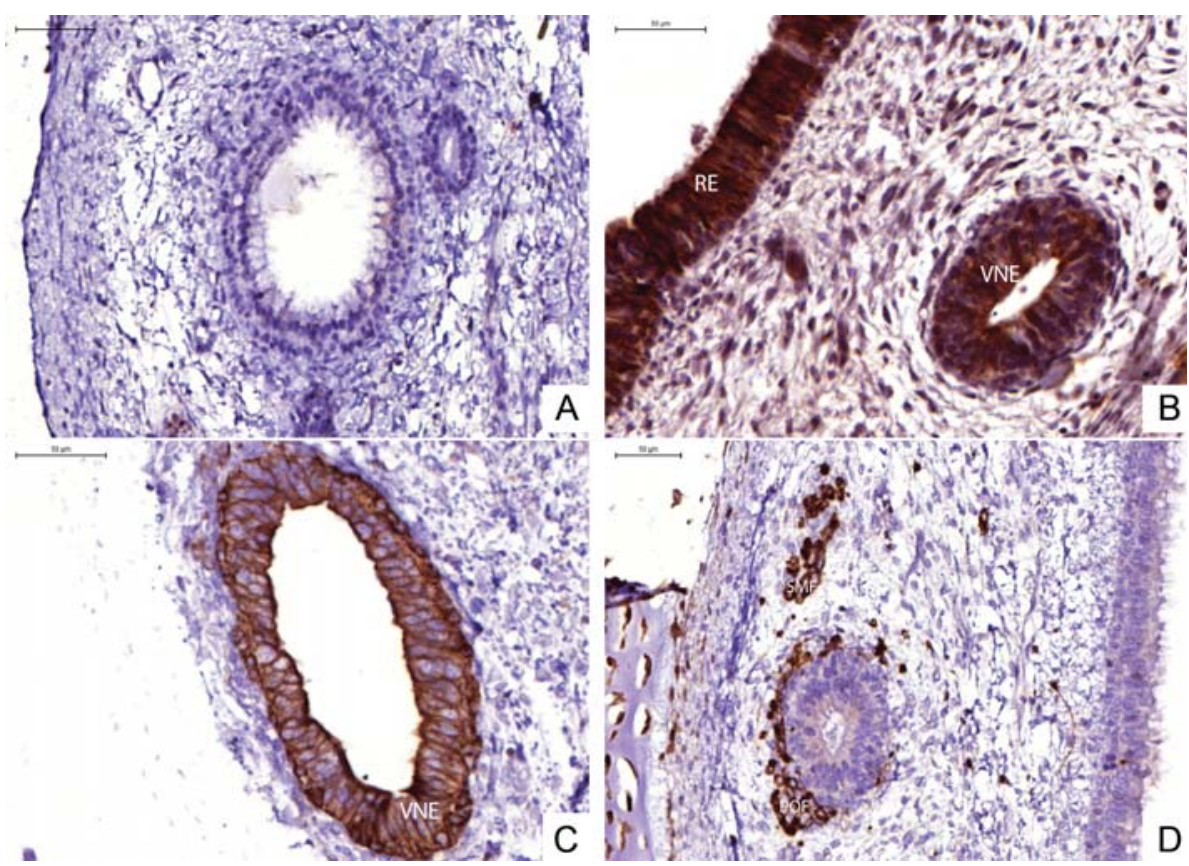

Figure 2. A) Neurofilament reaction in the VNO of the second age group, $\times 20$ magnification (Note the completely negative reaction).

B) Aspect of Ctk7 reaction in the first age group, $\times 20$ magnification

(RE - respiratory epithelium, with almost complete immunopositivity,

VNE - vomeronasal epithelium, with immunopositivity localized especially to the superior layers).

C) Pan-Ctk reaction in the VNO of the first age group,

$\times 40$ magnification (almost all cells are positive, underlining their epithelial origins).

D) Aspect of the $\mathrm{S} 100$ protein reaction in the first age group,

$\times 20$ magnification ( $\mathrm{POF}$ - periorgan fibres, positive nerve bundles adjacent to the VNO; there are also other positive nerve fibres bundles in the submucosa)

tribution described for group 1. There are a few intensively positive cells that sometimes reach the surface of the epithelium. The results of surface area measurements (Table IV) demonstrate a descending trend as foetal age increases. In case of smaller foetuses there is a fairly high percent of immunopositivity that shows almost continuous reduction. Foetuses included in group 3 show only very reduced, sometimes inexistent immunopositivity.

\section{Calretinin}

In the first and second age groups very intense reactions are observed, showing variations in the density of labelled cells. These cells are either found isolated in a small area, or they cluster in small groups of 4-5 cells. Vertical lo- 
Table IV. Average immunopositivity ratios of selected markers for the three age groups

\begin{tabular}{lcccc}
\hline \multirow{2}{*}{ Fetal age group } & \multicolumn{4}{c}{ Surface area of immunopositive cells (\%) } \\
\cline { 2 - 5 } & NSE & Calret & Ctk7 & PanCtk \\
\hline Group 1 & 21.81 & 17.97 & 22.69 & 49.53 \\
Group 2 & 10.59 & 13.52 & 60.77 & 47.75 \\
Group 3 & 0.64 & 0.75 & 57.50 & 50.94 \\
\hline
\end{tabular}

calization is variable, with groups seen on the basal membrane, in the intermediate layers and superior layers of the epithelium. Additionally, there are a few cell groups that extend over the whole thickness of the epithelium. Nevertheless, most of the positive cells are grouped on the basal membrane, and only isolated cells are seen in higher layers. The reaction is also positive for the nerve fibres of the submucosa that apparently decrease in numbers towards inferior areas of the nasal septum (Fig. 1C). The immunolabelling in the olfactory epithelium shows fundamental differences compared to the VNO (Fig. 1D). The positive reaction is extremely consistent and confined to bipolar olfactory cells, with axons crossing the basal membrane, resulting in a very orderly aspect of the reaction. Another variant of positive Calret pattern is a continuous layer of positive cells just above the basal layer, with apical processes extending towards the luminal surface, but lacking basal continuity. In the third age group Calret immunopositivity has disappeared almost completely. There are a few intensively immunopositive cells, but these are isolated in dispersed in the epithelium. Data collected by measuring the changes of Calret immunoexpression over time (based on foetal age) show variations of this marker, but there is a descending trend to be seen, similar to the previous marker (Table IV). In the first and second age group there are some changes, with a good immunopositivity still maintained, but in the third group positive reactions disappear almost completely.

Neurofilament, chromogranin, synaptophysin

NF reactions are negative in all three age groups. The only exceptions are a number of NF positive nerve fibres that are seen in the submucosa of the nasal cavity (Fig. 2A).

Just like for the previous marker, CHR reaction is negative for all age groups.

Synaptophysin shows negative immunoreactivity in the VNO and in all tissues of the foetal nasal cavity. In the first age group there are small areas of positive reaction in the vicinity of the $\mathrm{VNO}$, but these are located at a certain 
distance from the basal membrane of the organ in the submucosa, which excludes them as having potential nervous connections with the organ.

\section{Epithelial markers}

\section{Cytokeratin 7}

In the first age group a positive reaction is seen located mainly to the superior layers of the epithelium. In this area devoid of nuclei the positive reaction is continuous, and it is concentrated to the apical part of the cells. A number of immunopositive structures emerge from these cells heading towards the basal membrane, but none of these reaches its target. Thus, a polarized aspect is seen here, where the basal layer is completely negative, the intermediate layers show partial immunopositivity, and the luminal area of the epithelium is intensively positive. This aspect is not seen in the respiratory epithelium, where an almost homogeneous immunoexpression is observed throughout the entire thickness of the epithelium, contrasting the VN epithelium (Fig. 2B). In the second age group the positive reaction extends in an almost uniform manner to the whole epithelium, also including its inferior compartments, with the exception of the basal cells. In the last age group the intensively positive reaction extends to the whole surface of the VN epithelium, with enhanced immunopositivity of the non-nucleated superficial layer. It is to be noted that basal cells are also positive here, suggesting transformation of most of the cells into epithelial type cells, without negative compartments suggesting the presence of other cell types. The number of $\mathrm{Ctk} 7$ positive elements in the $\mathrm{VN}$ epithelium shows a gradual increase with advancement of foetal age (Table IV).

\section{Pan-cytokeratin}

In the first age group a positive reaction is seen extending throughout the whole epithelial surface, from the apical side and including the basal cells as well. The aspect is different from Ctk7, as it contours all cellular layers of the epithelium, providing a positive reaction at the periphery of the cytoplasm. Apparently there are no negative spaces in the VN epithelium, suggesting the epithelial origin of all cells making up the organ (Fig. 2C). Nevertheless, there are differences compared to the respiratory epithelium where the characteristic pseudostratified structure is visible, while in the VNO the outlined cells are larger, and seem to be organized into less overlying layers. In the next age group there is a strong immunopositivity in the basal cells, and in the non-nucleated apical part of the epithelium. In the intermediate layers the positive reaction is less intense, but still 
present in the outer cytoplasm of these cells. In case of the last age group there is a strong and generally uniform positive reaction extending throughout the whole epithelium. Overall, PanCtk immunopositivity does not show significant variations during the examined foetal period (Table IV).

Glial marker - S100 protein

No measurements were performed for this marker, since it was not expected to label any epithelial structures. There is positive immunoexpression localized close to different structures of the submucosa, and also to well define nerve bundles. Positive reaction is seen around certain exocrine glands of the nasal submucosa. In the first age group, immunopositive structures surround partially the medial aspect of the VNO, in close contact with its outer contour. At the superior pole of the organ there is a characteristic, annular immunopositivity (Fig. 2D). S100 reaction in the submucosa of the olfactory epithelium displays immunopositivity characteristic to olfactory nerve fibres, providing connections that pass the basal membrane into the epithelium. In case of the further age groups there is a decrease of the immunoexpression surrounding the VNO; in the last group only a few dispersed and isolated patches of positivity are observed in its vicinity.

\section{Discussions}

The fact the VNO occurred in less than half of the examined specimens is highly suggestive for its inconstant presence, which in turn raises questions about the phylogenetic significance of the organ for the human species.

Immunohistochemical research demonstrated that NSE is confined to neurons, [7] and not only to central neurons, but to neurons of spinal ganglia, autonomic neurons and certain peripheral neuroendocrine cells. Several reports have described that molecules specific to neurons can be localized using immunohistochemical methods to cells found in the VN epithelium of human foetuses $[1,8,9]$.

In the present work immunohistochemistry has been performed on several specimens of different age, in order to describe the time-dependent changes of the neural properties of the VN epithelium. The results obtained by NSE immunohistochemistry clearly demonstrate that in small foetuses there are cell populations, which can be considered to be neurons or neuroendocrine cells. In the context of respiratory epithelium and olfactory epithelium immunohistochemistry, there are major differences to be noted. Consequently, interpretation of the results has to bear in mind that immunoexpression in the VNO resembles most 
the pattern seen in the respiratory epithelium, which may contain neuroendocrine cells. However, several authors suggested that there is a well-represented neuron population up to the age of 5 foetal months, followed by a replacement period of these neurons by other cell types. This is also confirmed by the connections of these cells with nervous fibres located next to the VNO, which has been demonstrated by the present work, too. Regardless of the type of cells expressing NSE in the VN epithelium, the relative amount of immunoexpression shows a decreasing trend with age, and in the last age group it is already insignificant. This information correlates with reports by other authors who demonstrated reduced immunoexpression in the VN epithelia of adult humans [9].

In the nervous system calretinin is expressed specifically and almost exclusively in neurons, but not in glial cells [10]. There are reports of calretinin immunoexpression in the sensory pathways of the auditory, visual or olfactory systems [11]. In the main olfactory system and the VN system calretinin has been detected in receptor cells, in different interneurons (granule and periglomerular cells) and in the mitral cells of the main and accessory olfactory bulb of adult mammals $[12,13,14]$. In case of the foetal human VNO, the results of the present work found a decrease in the number of calretinin positive cells with age, which confirms the findings of the previous marker, NSE. Calretinin immunoexpression confirms the neural properties of these cells. Their initial abundance suggests that there is a differentiation potential of the VN epithelium towards a chemosensory epithelium. The existence of connections with immunolabelled structures resembling nervous fibres, located around the organ is also confirming this hypothesis. Finally, although at early stages there is a confirmed neuron population, in later stages of development this population diminishes, and it is taken over by cells without neural properties.

Neurofilaments start to appear in the central and peripheral nervous system of the mouse after days 9-10 of embryonic development, which corresponds to the time of initial axonal expansion [15]. These filaments are characteristic to differentiated neurons, and they replace vimentin filaments in the cytoskeleton of dividing neural precursors. Both types of intermediate filaments may be coexpressed for a short time [16]. In our study the presence of NF could not be confirmed in any of the examined specimens. Lack of a major component of neurons raises major questions regarding the capability of the VN epithelium to develop and differentiate into a neuroepithelium.

Chromogranin A is present in neuroendocrine cells throughout the body. It is the standard immunohistochemistry marker for neuroendocrine tumours, and increased levels of chromogranin $\mathrm{A}$ in the blood are diagnostic for these tumours [17]. In the context of positive NSE and Calret immunoexpression in early stages of foetal development, the lack of chromogranin immunoexpression 
suggests the existence of an initial population of precursors with neural properties, and without neuroendocrine elements, at least during the examined foetal period.

Usually associated to the membranes of synaptic vesicles, [18] synaptophysin has also been detected in sensory nerve endings of the peripheral nervous system. Synapsin I and synaptophysin are co-localized in the annulospiral sensory nerve endings of the muscle spindle, [19] and in calyciform sensory endings of vestibular receptors, [20] while synapsin I has been localized in the sensory endings of taste buds [21]. Negative reactions in both the VN and respiratory epithelium further underline the lack of production and storage of biomolecules required for information transmission.

Cytokeratin 7 is a fundamental cytokeratin found in most glandular epithelia, but it is not expressed by keratinized stratified epithelia. It is also expressed in the epithelial cells of the ovary, lung and breast, but not in the prostate or digestive tract [22].

Considering that $\mathrm{Ctk} 7$ labels the respiratory epithelium, it is interesting to follow the changes over the examined foetal period. Initially there is a Ctk7negative cell population, which correlates with the neural marker reactions. Subsequent extension of Ctk7 immunoexpression over the entire VN epithelium is suggestive for the transformation of neural elements into epithelial-like cells.

Use of the AE1/AE3 anti-cytokeratin cocktail (Pan-Ctk) has been considered in order to extend the possibilities to identify all cell populations with epithelial properties. Cytokeratins are considered supplemental markers to aid in differentiation of non-sensory cells from sensory cells in the olfactory epithelium $[23,24]$ and the VN epithelium [5]. While horizontal basal cells of the olfactory epithelium usually show intense immunoexpression for cytokeratin [24, 25] our results regarding the $\mathrm{VN}$ epithelium do not show a basal layer comparable to the one found in the olfactory epithelium, neither lacunae of negative reactions that might indicate the presence of chemosensory cells [26]. Pan-Ctk results of the present study demonstrate that during the studied foetal age intervals almost all VN epithelial cells display structural components associated to cytokeratins, suggesting the gradual transformation of putative neural precursors into epithelial cells.

S100 protein is normally expressed by cells developing from the neural crest, like Schwann cells, melanocytes, glial cells, and other cells like chondrocytes, myoepithelial cells, Langerhans cells [27]. No S100 protein (as glial cell marker) immunoexpression could be demonstrated in the vicinity of the adult VNO. However, S100 protein immunoexpression was showed in the vicinity of the olfactory epithelium in olfactory nerve fibres leaving the epithelium [5]. In our study we found nerve fibre bundles ensheathed by S100 positive glial cells 
in early foetal stages, but there was no evidence of contact with the VN epithelium. Additionally, in later stages these bundles became less evident. The above statements are confirmed by the S100 immunoexpression in the vicinity of the olfactory epithelium (used as internal control), where connections with olfactory nerve fibres could be clearly demonstrated.

Immunologically there are several aspects to be considered. The antibodies used in our study were developed for use in adult human tissues, which enabled us to detect the first occurrence of fully differentiated cell types, at least in the foetuses of the lowest age. Secondly there are changes in the distribution of immunopositivity over time, which demonstrated a shift in the immunological properties and epitope display of affected cells.

Sugata et al. [28] used antibodies directed against NSE and NF protein in the olfactory mucosa of human foetuses. They found weak NSE immunoreactivity in the olfactory receptor cells of the smaller age foetuses, and strong immunopositivity in older foetuses, and concluded that this pattern underlines the commitment of receptor cells towards neural differentiation. The present study described an inverse pattern of immunostaining, demonstrating the loss of this characteristic protein, and transformation of the initial neuron-like population. We found initial NSE positivity as early as the $9^{\text {th }}$ foetal week, while the abovementioned authors describe it at week 12 . This demonstrates the early presence of neuron-like immunological properties and at least partial maturation of these cells. Sugata et al. had seen NF immunopositivity only in older foetuses (week 16 and above), mentioning that this may be due to the lack of development of NF synthesis. They had seen NF positivity in the nerve fibers in the underlying mucosa as well. As we could not demonstrate any NF positivity in the VN epithelium, it seems likely that the initial neuron-like morphology of putative receptor cells never reaches full maturation.

\section{Conclusions}

The VNO is inconstantly detected during foetal development, and its presence is not conditioned by the gender or the age of the foetus. The VN epithelium initially contains a population of cells with neural properties, and displays a potential to differentiate into a chemosensory epithelium, but the neural population diminishes dramatically with advancement of foetal age. In spite the initially well-represented neural population, there are no evident connections with neighbouring nerve fibres. Negative immunoexpression of chromogranin and synaptophysin exclude the possible explanation of neuronal marker immunopositivity with the existence of neuroendocrine cells. Epithelial markers demon- 
strate the presence of cell types with different cytoskeletal components, but they become predominant in later stages, indirectly confirming the disappearance of nervous structures.

The results of the present work uphold the theory of regression of the neuroepithelium that is present during initial stages of foetal development.

\section{Acknowledgements}

The project has been funded by the Internal Research Grants of the University of Medicine and Pharmacy, Tîrgu Mureş, Romania (contract no. 32/16435/ 11.12.2013). Ethical permission number is UMFTGM-REG-74-F03.

\section{Conflict of Interest}

None. The authors have no conflict of interest.

\section{References}

1. Boehm, N., Gasser, B.: Sensory receptor-like cells in the human foetal vomeronasal organ. Neuroreport 4, 867-870 (1993).

2. Boehm, N., Roos, J., Gasser, B.: Luteinizing hormone-releasing hormone (LHRH)-expressing cells in the nasal septum of human fetuses. Developmental Brain Research 82, 175-180 (1994).

3. Kjaer, I., Fischer-Hansen, B.: The human vomeronasal organ: prenatal developmental stages and distribution of luteinizing hormone-releasing hormone. European Journal of Oral Sciences 104, 34-40 (1996).

4. Smith, T., Siegel, M., Mooney, M., Burdi, A., Burrows, A., Todhunter, J.: Prenatal growth of the human vomeronasal organ. Anatomical Record 248, 447-455 (1997).

5. Trotier, D., Eloit, C., Wassef, M., Talmain, G., Bensimon, J.L., Døving, K.B., Ferrand, J.: The vomeronasal cavity in adult humans. Chem Senses 25, 369-380 (2000).

6. Sadler, T.W.: Langman's Medical Embryology, 10th Edition. Lippincott Williams \& Wilkins, 2006, p. 118

7. Pickel, V.M., Reis, D.J., Marangos, P.J., Zomzely-Neurath, C.: Immunocytochemical localization of nervous system specific protein (NSP-R) in rat brain. Brain Res 105, 184 187 (1976).

8. Johnson, E.W., Eller, P.M., Jafek, B.W.: Calbindin-like immunoreactivity in epithelial cells of the newborn and adult human vomeronasal organ. Brain Research 638, 329-333 (1994).

9. Takami, S., Getchel, M.L., Chen, Y., Monti-Bloch, L., Berliner, D.L., Stensaas, L.J. et al.: Vomeronasal epithelial cells in the adult human express neuron-specific molecules. Neuro-report 4, 375-378 (1993). 
10. Rogers, J.H.: Calretinin: a gene for a novel calcium-binding protein expressed principally in neurons. J Cell Biol 105, 1343-1353 (1987).

11. Jacobowitz, D.M., Winsky, L.: Immunocytochemical localization of calretinin in the forebrain of the rat. J Comp Neurol 304, 198-218 (1991).

12. Malz, C.R., Knabe, W., Kuhn, H.J.: Pattern of calretinin immunoreactivity in the main olfactory system and the vomeronasal system of the tree shrew, Tupaia belangeri. J Comp Neurol 420, 428-436 (2000).

13. Alonso, J.R., Briñón, J.G., Crespo, C., Bravo, I.G., Arévalo, R., Aijón, A.: Chemical organization of the macaque monkey olfactory bulb: II. Calretinin, calbindin D-28k, parvalbumin, and neurocalcin immunoreactivity. J Comp Neurol 432, 389-407 (2001).

14. Briñón, J.G., Weruaga, E., Crespo, C., Porteros, Á., Arévalo, R., Aijón, A., Alonso, J.R.: Calretinin-, neurocalcin-, and parvalbumin immunoreactive elements in the olfactory bulb of the hedgehog (Erinaceus europeus). J Comp Neurol 429, 4-50 (2001).

15. Cochard, P., Paulin, D.: Initial expression of neurofilaments and vimentin in the central and peripheral nervous system of the mouse embryo in vivo. J Neurosci 4, 2080-2094 (1984).

16. Tapscott, S.J., Bennett, G.S., Holtzer, H.: Neuronal precursor cells in the chick neural tube express neurofilament proteins. Nature (Lond.) 282, 853-855 (1981).

17. O'Connor, D.T., Deftos, L.J.: Secretion of chromogranin A by peptide-producing endocrine neoplasms. N Engl J Med 314, 1145-1151 (1986).

18. De Camilli, P., Jahn, R.: Pathways to regulated exocytosis in neurons. Annu Rev Physiol 52, 625-645 (1990).

19. De Camilli, P., Vitadello, M., Canevini, M.P., Zanoni, R., Jahn, R., Gorio, A.: The synaptic vesicle proteins synapsin I and synaptophysin (protein 38) are concentrated both in efferent and afferent nerve endings of the skeletal muscle. J Neurosci 8, 1625-1631 (1988).

20. Scarfone, E., Dememes, D., Jahn, R., De Camilli, P., Sans, A.: Secretory function of the vestibular nerve calyx suggested by presence of vesicles, synapsin I and synaptophysin. J Neurosci 8, 4640-4645 (1988).

21. Finger, T.E., Womble, M., Kinnamon, J.C., Ueda, T.: Synapsin I-like immunoreactivity in nerve fibers associated with lingual taste buds of the rat. J Comp Neurol 292, 283-290 (1990)

22. Sun, T.T., Skelton, H.G., Green, H.: Keratin cytoskeletons in epithelial cells of internal organs. Proc Nat Acad Sci U S A 76, 2813-2817 (1979).

23. Okabe, H., Okubo, T., Adachi, H., Ishikawa, T., Ochi, Y.: Immunohistochemical demonstration of cytokeratin in human embryonic neurons arising from placodes. Brain Dev 19, 347-352 (1997).

24. Satoh, M., Yoshida, T.: Expression of neural properties in olfactory cytokeratin-positive basal cell line. Brain Res Dev Brain Res 121, 219-222 (2000).

25. Holbrook, E.H., Szumowski, K.E., Schwob, J.E.: An immunochemical, ultrastructural, and developmental characterization of the horizontal basal cells of rat olfactory epithelium. J Comp Neurol 363, 129-146 (1995).

26. Witt, M., Georgieva, B., Knecht, M., Hummel, T.: On the chemosensory nature of the vomeronasal epithelium in adult humans. Histochem Cell Biol 117, 493-509 (2002).

27. Schäfer, B.W., Heizmann, C.W.: The S100 family of EFhand calcium-binding proteins: functions and pathology. Trends Biochem Sci 21, 134-140 (1996).

28. Sugata, T., Toshihiko, I., Yasuo, T., Yuichi, N., Tsuneo, F.: Neuron-specific enolase, neurofilament protein and S-100 protein in the olfactory mucosa of human fetuses. Cell and Tissue Research 238, 231-234 (1984) 